\title{
Variação dos valores da distância percorrida e da velocidade de deslocamento em sessões de treino e em competições de futebolistas juniores
}

\author{
Pedro F. Caixinha ${ }^{1}$ \\ Jaime Sampaio ${ }^{2}$ \\ Pedro V. Mil-Homens ${ }^{3}$
}

https://doi.org/10.5628/rpcd.04.01.07

\section{RESUMO}

O presente estudo teve como objectivo a comparação dos valores da distância percorrida, da velocidade e do tempo de acção medidos nas sessões de treino e nas competições de Futebol. A amostra utilizada foi composta por 3 jogadores juniores (19 anos) que foram observados em 3 microciclos de treino e 3 competições. Durante o período de observação, estes desportistas encontravam-se a disputar a $1^{\text {a }}$ fase do Campeonato Nacional (época 2002/2003). Desempenharam na equipa diferentes posições, nomeadamente de defesa central (DC), médio-centro (MC) e ponta-de-lança (PL). Para medir as coordenadas e o tempo foi utilizado o software Tacto. Os resultados obtidos sugeriram diferenças estatisticamente significativas entre os valores obtidos nas sessões de treino comparativamente com os valores obtidos na competição. $\mathrm{O}$ jogador $\mathrm{MC}$ foi o que maior distância percorreu em situação de treino $(10309 \mathrm{~m})$ e de competição $(14385 \mathrm{~m})$. Os valores dos restantes jogadores foram os seguintes para o treino e competição: (DC) $8637 \mathrm{~m}$ e $13374 \mathrm{~m}$ e (PL) $9560 \mathrm{~m}$ e $13355 \mathrm{~m}$. Concomitantemente, os jogadores atingiram valores inferiores de velocidade de deslocamento no treino relativamente aos valores da competição, com a excepção do DC (DC: $2,7 \mathrm{~m} / \mathrm{s}$ vs. $2,4 \mathrm{~m} / \mathrm{s}$; MC: $2,4 \mathrm{~m} / \mathrm{s}$ vs. $2,5 \mathrm{~m} / \mathrm{s}$; PL: 2,3 vs. $3,6 \mathrm{~m} / \mathrm{s})$. No contexto do treino, os valores da velocidade de deslocamento foram superiores na $2^{\mathrm{a}}$ parte do treino, enquanto que em competição os valores foram superiores na $1^{a}$ parte do jogo. Na generalidade, os resultados sugeriram que $o$ jogo de Futebol solicitou aos jogadores componentes da aptidão física diferentes das solicitadas nas sessões de treino, pelo que se pode dizer que o trabalho observado nas sessões de treino foi menos específico do que seria desejável.

Palavras-chave: Futebol, distância percorrida, velocidade de deslocamento, treino, competição, jovens.
${ }^{1}$ Comissão Instaladora dos Ensinos na Área da Saúde e do Bem Estar, Universidade de Évora, Portugal

${ }^{2}$ Departamento de Desporto, Universidade de Trás-os-Montes e Alto Douro, Portugal

${ }^{3}$ Faculdade de Motricidade Humana da Universidade Técnica de Lisboa, Portugal

\section{ABSTRACT \\ Speed and distance covered during training sessions and competi- tions of youth soccer players.}

The aim of this study was to compare distance covered, speed and action time in soccer, players during training and competition. The sample comprised 3 junior male soccer players (aged 19 years old) observed during 3 sets of training units and 3 competitions of the $1^{\text {st }}$ phase of the Portuguese National Championship. Players' positions were as follows: DC - defence, $M C$ - midfield and PL - striker. Coordinates and time were evaluated according to software Tacto. Results suggested statistical significant differences for all variables between training and competition. Midfield player covered more distance during training sessions $(10309 \mathrm{~m})$ and during competitions $(14385 \mathrm{~m})$. The rest of the players' results were: (DC) $8637 \mathrm{~m}$ versus $13374 \mathrm{~m}$ and $(P L) 9560 \mathrm{~m}$ versus $13355 \mathrm{~m}$. Conversely, players' speed during training sessions was lower than during competitions, exception made to DC (DC: $2,7 \mathrm{~m} / \mathrm{s}$ vs. $2,4 \mathrm{~m} / \mathrm{s} ; M C: 2,4 \mathrm{~m} / \mathrm{s}$ vs. $2,5 \mathrm{~m} / \mathrm{s}$; PL: 2,3 vs. 3,6 m/s). During training sessions speed results were higher in the $2^{\text {nd }}$ part, whereas in competition they were higher in the $1^{\text {st }}$ part of the game. These results suggested that soccer game elicited to the players' different fitness components than those who were elicited during training sessions. This observation suggested that physical demands during the observed training sessions were less specific than those expected by game characteristics.

Key Words: Soccer, distance covered, speed, training, competition, youth. 


\section{INTRODUÇÃO}

A literatura disponível acerca da actividade motora desenvolvida pelos jogadores de Futebol direccionase, quase exclusivamente, para a caracterização do perfil energético-funcional dos jogadores, imposto pela configuração estrutural e funcional da competição. Os caminhos mais percorridos para a procura destas respostas têm procurado caracterizar indicadores externos, i.e., as variáveis mais estudadas têm sido as distâncias totais e as distâncias parciais (tipo, duração, frequência e intensidade dos deslocamentos) e a relação entre os tempos (totais e parciais) de actividade e de pausa (9). De facto, a caracterização das exigências físicas da competição, através dos designados estudos de tempo e movimento tem permitido identificar o número, tipo e frequência das acções realizadas pelo jogador $(4,9)$.

Particularmente no que se refere às distâncias percorridas, a literatura parece consensual. Apesar de serem várias as metodologias utilizadas, nos últimos 20 anos a distância média percorrida durante um jogo tem-se mantido entre os 8000 e $12000 \mathrm{~m}$ (1, 14, 17, 23). A variação intra-individual da distância percorrida por jogo é relativamente pequena e consistente; Bangsbo et al. (1) encontraram uma variação de $920 \mathrm{~m}$ ou $8,5 \%$.

$\mathrm{Na}$ maioria dos estudos realizados, verifica-se que os médios-centro percorrem maiores distâncias, enquanto que os defesas centrais são os que percorrem menores distâncias e efectuam grande percentagem destas distâncias de costas ou de lado (1, 7, 16). O estudo de Ekblom (7) é orientador neste domínio. Os resultados obtidos evidenciaram que os médios-centro percorrem mais $5 \%(10600 \mathrm{~m})$ do que o resto da equipa e que os avançados e os defesas percorreram $10100 \mathrm{~m}$ e $9600 \mathrm{~m}$, respectivamente. $\mathrm{O}$ autor refere ainda que os resultados tiveram uma variação inter-jogos muito reduzida (9100-9600m para os defesas, $10200-11100 \mathrm{~m}$ para os médios-centro e $9800-10600 \mathrm{~m}$ para os avançados). Para complementar estes dados, Bangsbo et al. (1) verificaram que os médios se encontravam parados em $14,4 \%$ do tempo total, enquanto que os valores dos defesas e avançados foram mais elevados $(21,7 \%$ e $17,9 \%$, respectivamente). Por outro lado, os autores também identificaram diferenças na distância percorrida a baixa velocidade, na qual os médios percorreram
$3730 \mathrm{~m}$, os defesas $2040 \mathrm{~m}$ e os avançados $2550 \mathrm{~m}$. Os estudos realizados no âmbito da avaliação e consequente caracterização do padrão da actividade motora no Futebol, apenas têm sido realizados em contexto de competição, excepção feita ao estudo de Luhtanen (10). Este é a única referência que encontramos disponível e que pretende comparar o perfil de actividade motora de um jogador de alto nível no treino e na competição. Os resultados obtidos permitiram verificar que a distância total percorrida por um jogador ao longo do treino foi idêntica à dos jogos (11200m vs. $12000 \mathrm{~m}$, respectivamente). Contudo e como seria de esperar face à frequência de cada uma das situações, os valores acumulados ao longo da época foram bem diferentes $(67200 \mathrm{~m}$ vs. $264000 \mathrm{~m}$, respectivamente para a competição e para o treino). Neste trabalho está omissa a descrição dos procedimentos utilizados para recolher os dados, facto que limita qualquer análise mais profunda. Face às questões anteriormente apresentadas, surgenos uma questão que parece merecer reflexão mais profunda: (i) será que existe alguma relação entre os indicadores externos medidos no treino e medidos na competição?

Como já foi referido, a literatura disponível é omissa relativamente a esta matéria. Ou seja, o facto de apenas estarem disponíveis, de forma mais consistente, estudos realizados no contexto da competição, conduz-nos à tentativa de esclarecer, através do estudo de indicadores externos, o relacionamento entre o esforço para o qual o atleta é preparado (esforço em treino) e o esforço ao qual o atleta é sujeito (esforço na competição). Mais ainda, se nos reportarmos às amostras seleccionadas para os referidos estudos, constatamos também que apenas são estudados os atletas seniores, ou seja, não existem dados disponíveis que caracterizem o padrão de actividade motora de futebolistas mais jovens. Adicionalmente, o estudo das diferenças entre os registos da $1^{\mathrm{a}}$ parte vs. $2^{\mathrm{a}}$ parte do treino e da competição poderá auxiliar na caracterização dos perfis de esforço já referidos. Neste sentido, o conjunto de informações que se pode retirar da resolução desta tarefa poderá constituir-se como um complemento fundamental para suportar as decisões dos treinadores relativamente à condução do processo de preparação desportiva das equipas, i.e., organizar o treino de acordo com a 
especificidade da competição. Em face do exposto e do conjunto de questões anteriormente colocadas, o presente estudo tem como objectivo a comparação dos valores da distância percorrida, da velocidade e do tempo de acção medidos nas sessões de treino e na competição numa amostra de jovens futebolistas.

\section{MATERIAL E MÉTODOS}

\section{Amostra}

A amostra foi constituída por 3 jogadores internacionais portugueses da categoria de juniores "A" (19 anos, altura $168,7 \pm 11,7$; peso $66,7 \pm 6,7$ e 9 anos de prática) que durante o período de observação se encontravam a disputar a $1^{\text {a }}$ fase do Campeonato Nacional da respectiva categoria (época 2002/2003). Desempenharam na equipa diferentes posições, nomeadamente de defesa central (DC), médio-centro (MC) e ponta-de-lança (PL).

\section{Procedimentos}

Foram registados em vídeo três microciclos de treino $(n=15)$ e três competições $(n=3)$. As sessões de treino foram divididas em duas partes iguais, a partir do tempo da parte principal (excluímos o aquecimento e o retorno à calma). Para as gravações em vídeo, foram utilizados e colocados sobre as linhas laterais do campo, dois elevadores eléctricos (cada um sobre a meia distância de cada meio campo) a $5,4 \mathrm{~m}$ da linha lateral e a $7,5 \mathrm{~m}$ de altura do solo. Em cada elevador foi colocada uma câmara de vídeo sobre um tripé. A recolha das imagens foi iniciada após o período de instrução inicial nos treinos e após o apito inicial do árbitro nos jogos. Em cada unidade de treino foram recolhidos dados de 2 jogadores da amostra, uma câmara acompanhava os movimentos de um jogador, ao passo que a outra seguia os movimentos do outro jogador. As imagens foram sempre recolhidas em ângulo aberto (sem alterações do zoom). Todos registos obtidos foram digitalizados para tratamento no software Tacto (8). Numa primeira fase, o utilizador acompanha todas as movimentações realizadas pelo jogador que está a analisar (com o recurso a um rato "sem fios") e o software calcula de imediato as coordenadas nos eixos $\mathrm{X}$ e $\mathrm{Y}$ da sequência em causa, que ainda não correspondiam aos valores reais, pois tinham que ser calibrados. Na calibração, registou-se também o número do campo onde foram recolhidas as imagens (uma vez que as distâncias reais dos campos utilizados pelas equipas eram diferentes), para de seguida e com a imagem parada, se proceder à calibração do campo em que o jogador se encontrava. Foi realizado um estudo piloto para confirmar a validade e a fiabilidade deste instrumento de medição (8). De forma a validar a posição real do jogador, comparámos a distância real e a distância calculada pelo programa, na realização de um percurso previamente estabelecido. Foram utilizadas diferentes velocidades de quadros de imagem por segundo (q/s) para o estudo de validação do instrumento $(1 \mathrm{q} / \mathrm{s}, 2 \mathrm{q} / \mathrm{s}, 10 \mathrm{q} / \mathrm{s}$ e $25 \mathrm{q} / \mathrm{s})$. Através da média absoluta da percentagem de erro $\left(\mathrm{M}_{\mathrm{E}}\right)$, comparámos as distâncias calculadas com as distâncias reais, $\mathrm{M}_{\mathrm{E}}=$ $100 / \mathrm{n} \Sigma\left|\mathrm{d}_{\mathrm{R}}-\mathrm{d}_{\mathrm{C}}\right| / \mathrm{d}_{\mathrm{R}}$, onde $\mathrm{n}=$ número de distâncias calculadas, $\mathrm{d}_{\mathrm{R}}=$ distância real e $\mathrm{d}_{\mathrm{C}}=$ distância calculada. Para medir a fiabilidade do instrumento, para o mesmo jogador e para os mesmos quadros de imagem, foi calculado o coeficiente de correlação intraclasse. As médias absolutas da percentagem de erro para as quatro distâncias calculadas foram: $\mathrm{M}_{\mathrm{EdC}(1 q / \mathrm{s})}=22.09 \% ; \mathrm{M}_{\mathrm{EdC}(2 q / \mathrm{s})}=18.62 \%$; $\mathrm{M}_{\mathrm{EdC}(10 \mathrm{q} / \mathrm{s})}=5.68 \% ; \mathrm{M}_{\mathrm{EdC}(25 \mathrm{q} / \mathrm{s})}=4.22 \%$. O coeficiente de correlação intraclasse foi de $\mathrm{r}=1.0$ assumindo o erro padrão de medida (SEM). Para a posição de $X: \operatorname{SEM}_{(1 \mathrm{q} / \mathrm{s}-25 \mathrm{q} / \mathrm{s})}=6.68 ; \operatorname{SEM}_{(2 \mathrm{q} / \mathrm{s}-25 \mathrm{q} / \mathrm{s})}=6.35$; $\operatorname{SEM}_{(10 \mathrm{q} / \mathrm{s}-25 \mathrm{q} / \mathrm{s})}=5.28$; e para a posição de $\mathrm{Y}$ : $\operatorname{SEM}_{(1 \mathrm{q} / \mathrm{s}-25 \mathrm{q} / \mathrm{s})}=5.40 ; \operatorname{SEM}_{(2 \mathrm{q} / \mathrm{s}-25 \mathrm{q} / \mathrm{s})}=5.80 ; \operatorname{SEM}_{(10 \mathrm{q} / \mathrm{s}-}$ $25 \mathrm{q} / \mathrm{s})=5.21$ e o factor médio de escala $(\xi)$ foi de $3,8 \mathrm{~cm}$, o que representa $\pm 22.5 \mathrm{~cm}$ de erro. Deste modo, concluímos que com a utilização de uma recolha e consequente tratamento de imagens a 25 $\mathrm{q} / \mathrm{s}$, o erro é inferior a $5 \%$.

\section{Análise dos resultados}

Para as comparações das distâncias percorridas e tempos de acção para cada um dos diferentes contextos (treino vs. competição) foi utilizado o teste de Mann-Whitney U. Nestas variáveis não foram comparados, do ponto de vista estatístico, os jogadores em função da sua posição. A variação da velocidade face à $1^{\mathrm{a}}$ ou $2^{\mathrm{a}}$ parte do jogo, ao contexto (treino vs. competição) e à posição dos jogadores (DC vs. MC vs. PL) foi analisada pelo recurso à Anova Factorial. A opção por esta técnica de análise estatística para- 
métrica justificou-se na medida em que o software de recolha de dados permitiu-nos obter um número de registos da velocidade instantânea dos jogadores muito elevado $(n=300617)$. Esta possibilidade não se verificou para as variáveis distância e tempo de acção porque o software apenas possibilita a recolha dos valores acumulados da distância e não os parciais. O nível de significância foi mantido em $5 \%$.

\section{RESULTADOS}

Os valores médios da distância total percorrida foram significativamente mais elevados em situação de competição ( $p<0.001$, ver Figura 1$)$. Na análise por posição, destacou-se o jogador MC como o que maior distância percorreu em situação de treino (10309m) e de competição (14385m). Os valores dos restantes jogadores foram os seguintes para o treino e competição: (DC) $8637 \mathrm{~m}$ e $13374 \mathrm{~m}$ e (PL) $9560 \mathrm{~m}$ e $13355 \mathrm{~m}$.

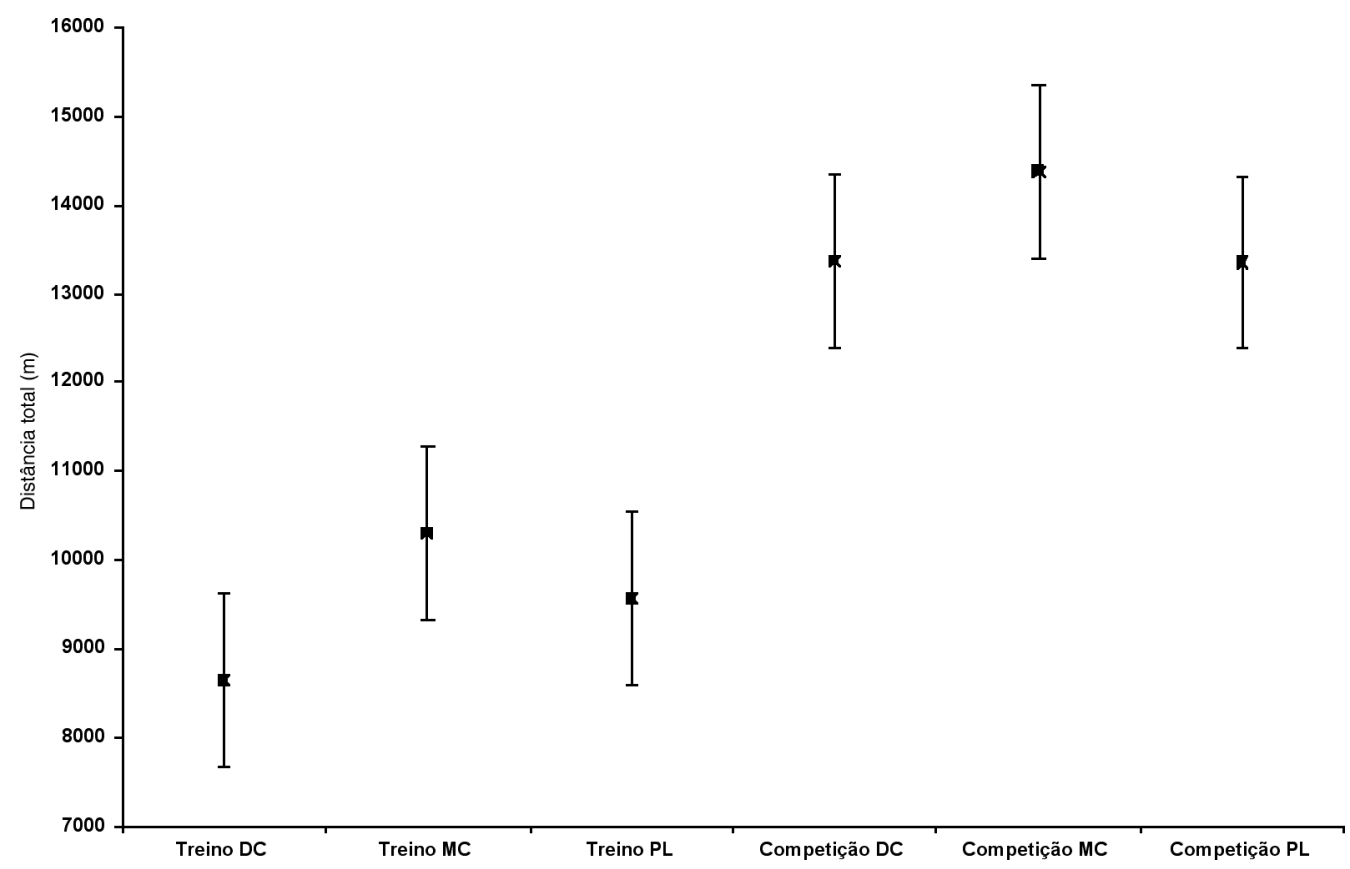

Figura 1. Variação dos valores médios da distância percorrida $(\mathrm{m}$ ] em função do contexto (treino vs. competição] e da posição dos jogadores (DC, $M C, P L$ ].

Os valores médios da velocidade também foram significativamente mais elevados em situação de competição (contexto x posição, $p<0.05$, ver Figura 2 ). $\mathrm{O}$ jogador com os valores mais elevados no contexto do treino foi o DC, enquanto que em competição foi o PL que obteve valores superiores. Os valores obtidos indicam que os jogadores atingem valores inferiores de velocidade no treino relativamente aos valores da competição, com a excepção do DC (DC: $2,7 \mathrm{~m} / \mathrm{s}$ vs. $2,4 \mathrm{~m} / \mathrm{s}$; MC: $2,4 \mathrm{~m} / \mathrm{s}$ vs. $2,5 \mathrm{~m} / \mathrm{s}$; PL: 2,3 vs. $3,6 \mathrm{~m} / \mathrm{s}$ ). Saliente-se no entanto que, no treino, a velocidade "diminuiu no sentido do ataque" $\left(\mathrm{V}_{\mathrm{DC}}>\mathrm{V}_{\mathrm{MC}}>\mathrm{V}_{\mathrm{PL}}\right)$, enquanto que em competição a velocidade "aumentou no sentido do ataque" $\left(\mathrm{V}_{\mathrm{DC}}<\right.$ $\left.\mathrm{V}_{\mathrm{MC}}<\mathrm{V}_{\mathrm{PL}}\right)$. 


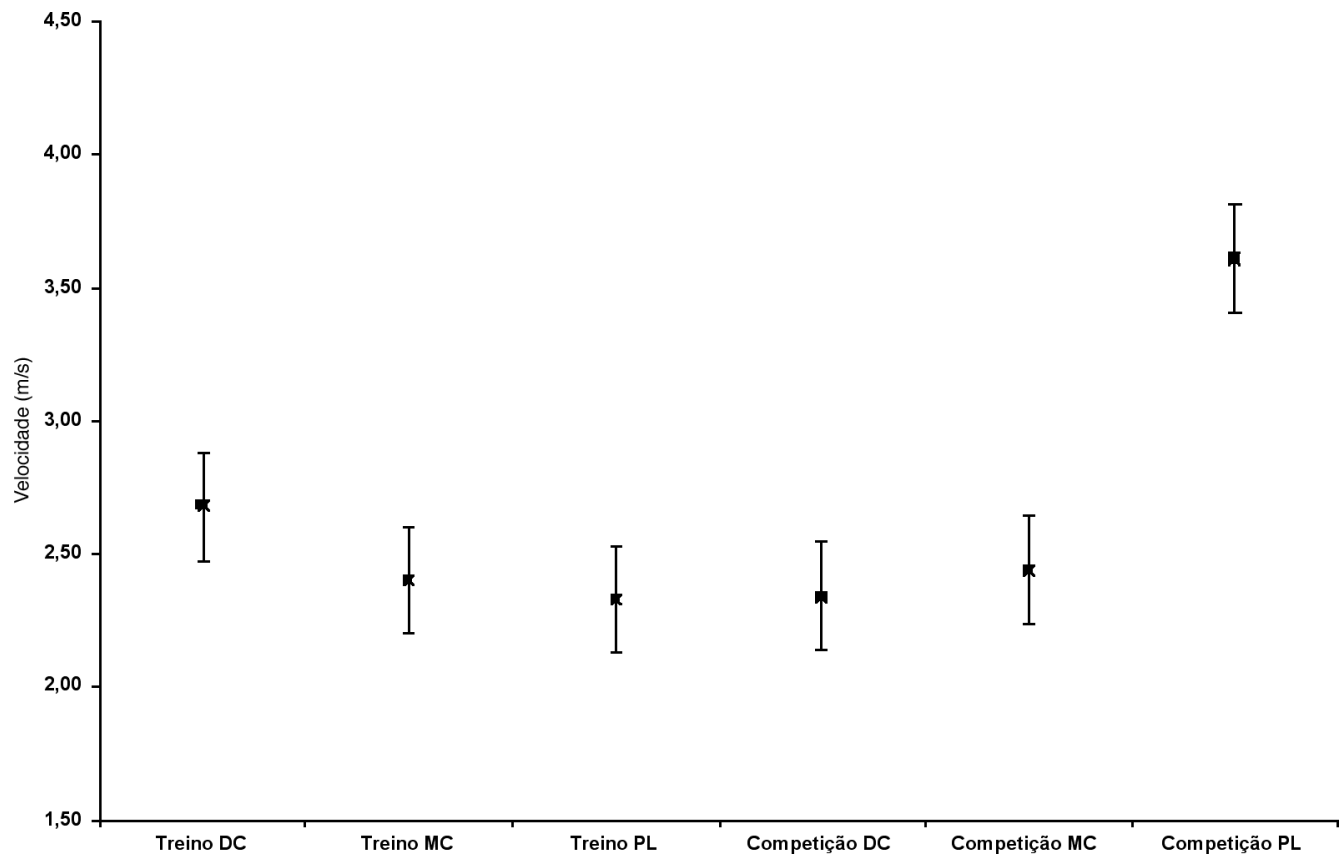

Figura 2. Variação dos valores médios da velocidade de deslocamento $(\mathrm{m} / \mathrm{s}$ ) em função do contexto (treino vs. competição] e da posição dos jogadores (DC, $M C, P L$ ].

Quando comparamos os valores da velocidade média dos jogadores entre a $1^{\text {a }}$ e a $2^{\mathrm{a}}$ parte do jogo nos dois contextos, os resultados identificaram um efeito estatisticamente significativo (contexto $\mathrm{x}$ parte, $p$ $<0.001)$ e apresentaram tendências inversas (ver Figura 3). No contexto do treino os valores foram superiores na $2^{\mathrm{a}}$ parte do treino, enquanto que em competição os valores foram superiores na $1^{\mathrm{a}}$ parte do jogo.

Figura 3. Variação da velocidade de deslocamento para a $1^{a}$ e $2^{a}$ parte relativamente aos diferentes contextos.

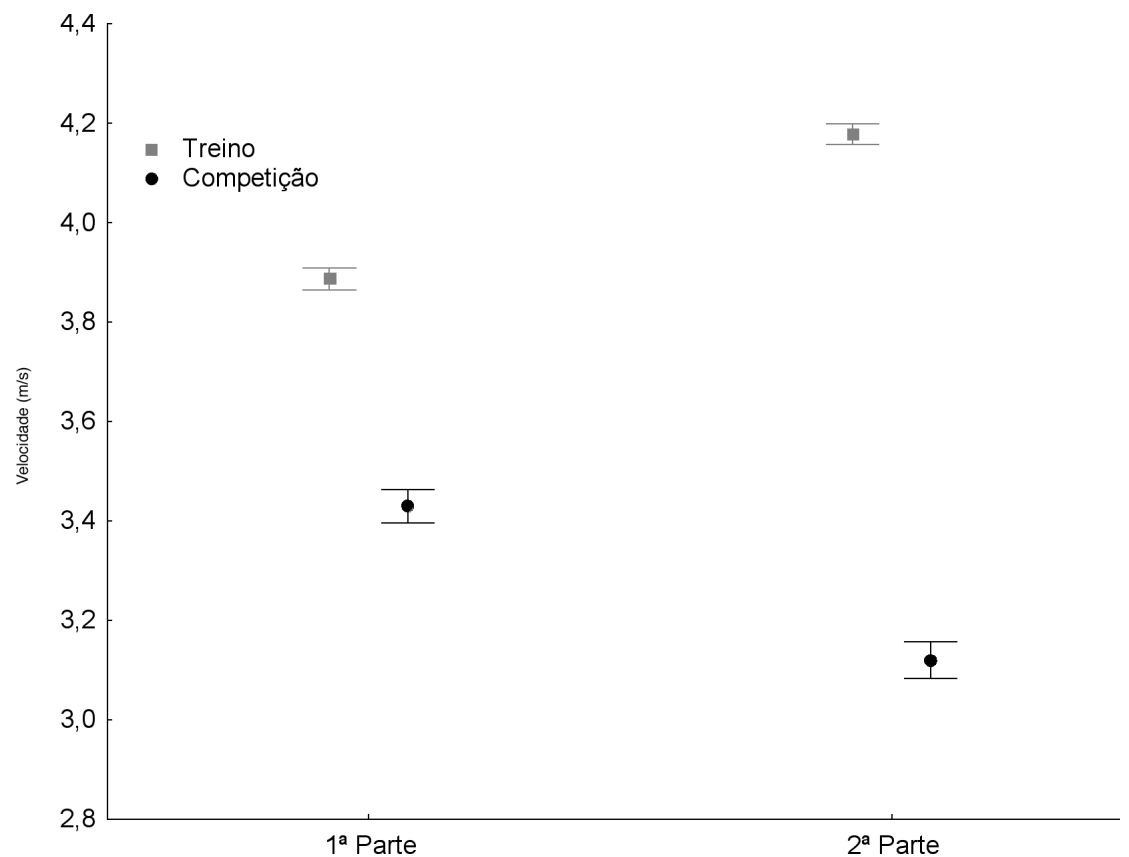




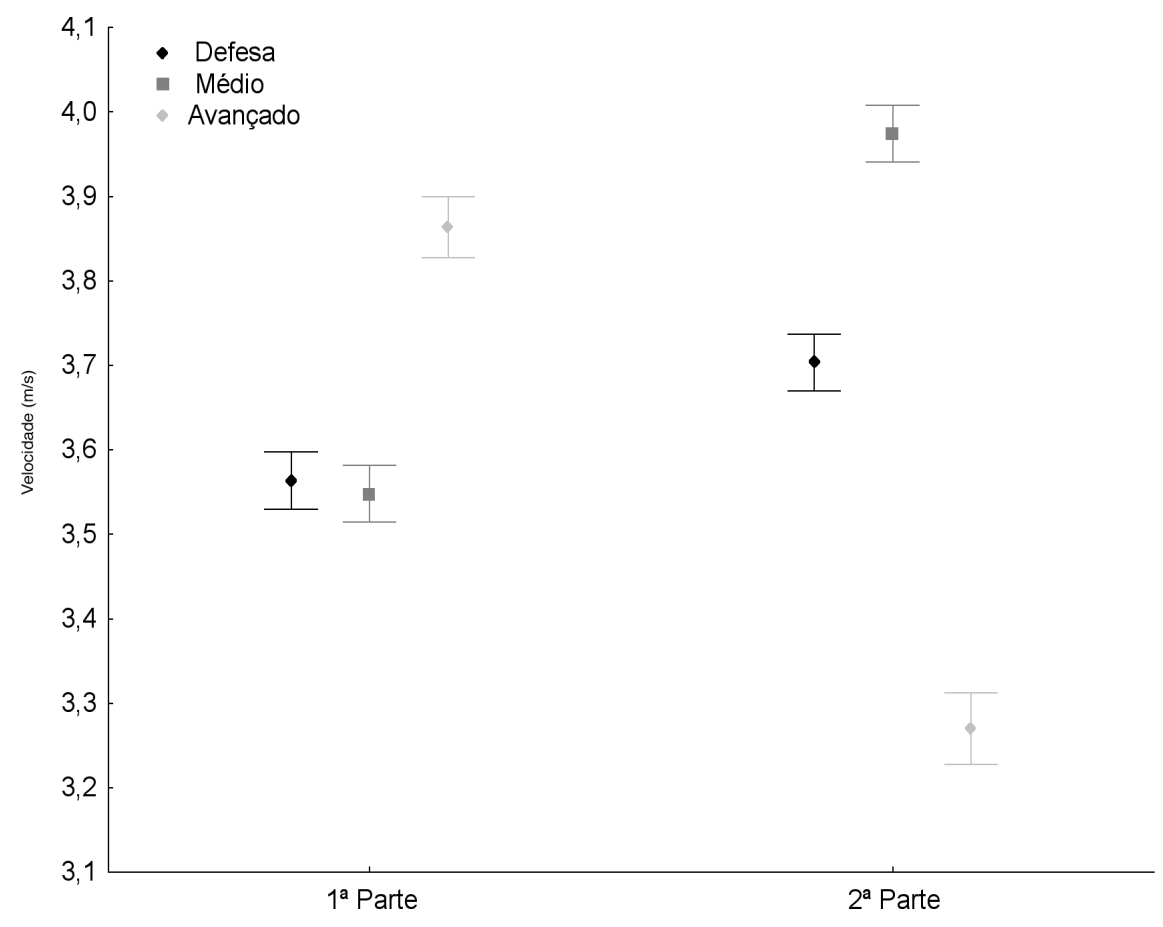

Relativamente à comparação entre os valores da $1^{\mathrm{a}}$ e da $2^{\mathrm{a}}$ parte do jogo em função da posição dos jogadores também foi identificado um efeito estatisticamente significativo (parte x posição, $p$ $<0.001$ ). Verificou-se que o DC e o MC apresentaram aumentos na velocidade média da $1^{\mathrm{a}}$ para a $2^{\mathrm{a}}$ parte, enquanto que o PL apresentou um decréscimo de velocidade da $1^{\mathrm{a}}$ para a $2^{\mathrm{a}}$ parte do jogo (ver Figura 4).

Figura 4. Variação da velocidade de deslocamento para a $1^{a}$ e $2^{a}$ parte relativamente às diferentes posições.

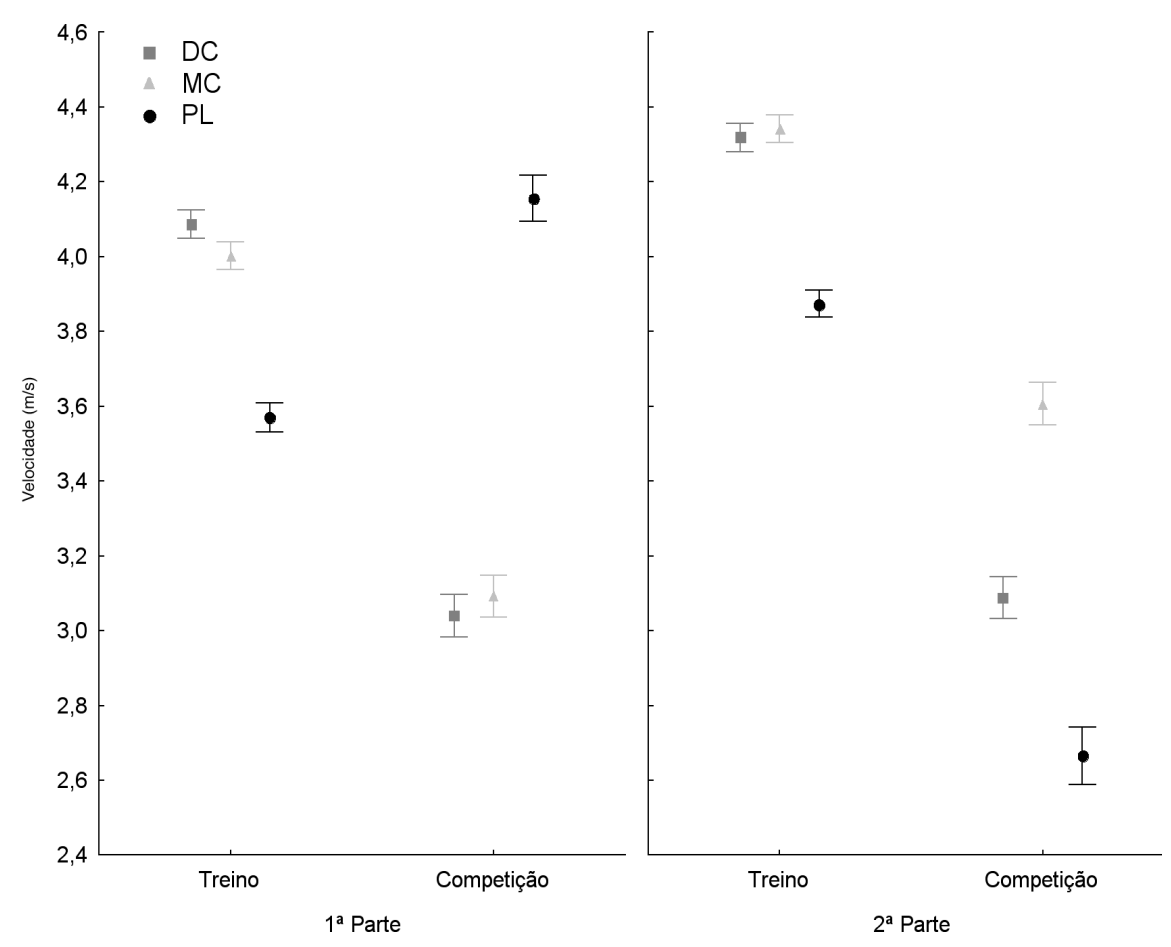

Os resultados da tripla interacção (contexto $\mathrm{x}$ parte $\mathrm{x}$ posição) também foram estatisticamente significativos $(p<0.001$, ver Figura 5), evidenciando a influencia dos três factores nos valores da velocidade.

Figura 5. Variação da velocidade de deslocamento para a $1^{a}$ e $2^{a}$ parte relativamente às diferentes posições e contextos. 
Finalmente, os valores médios do tempo de acção dos jogadores também foram significativamente mais elevados em situação de competição $(p<0.05$, ver Figura 6). O PL atingiu os valores mais elevados, ou seja, teve mais tempo de acção no treino e o MC foi o jogador que teve mais tempo de acção em competição. Os valores obtidos, em minutos, respectivamente para o treino e para a competição foram os seguintes: DC, 61' vs. 95'; MC, 70' vs. 98'; PL, 70' vs. 72 '.

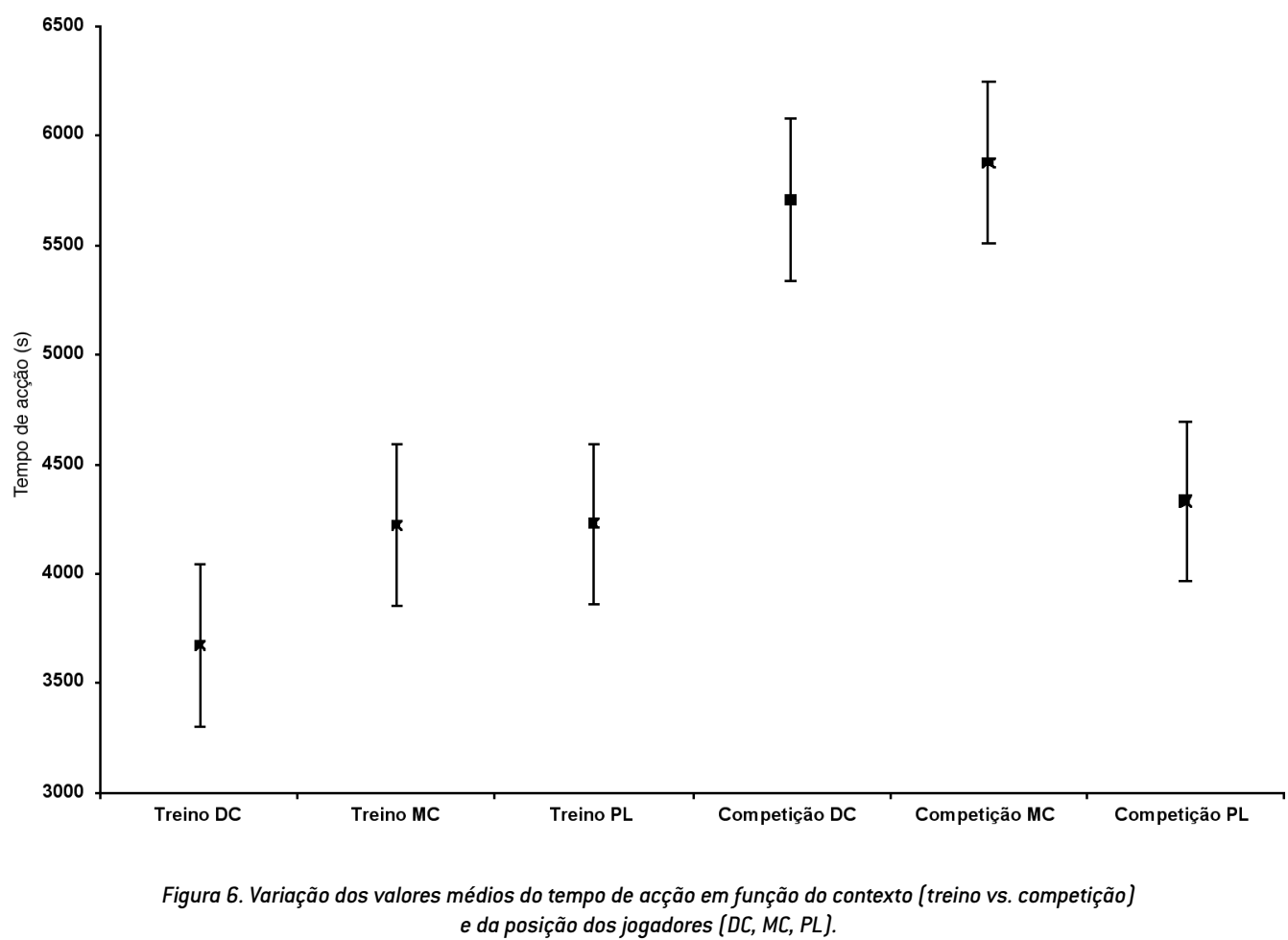

\section{DISCUSSÃO}

O objectivo do presente estudo foi o de comparar os valores da distância percorrida, da velocidade e do tempo de acção medidos nas sessões de treino e na competição numa amostra de jovens futebolistas. Numa primeira análise, os resultados obtidos sugerem diferenças substanciais entre os valores obtidos nas sessões de treino comparativamente com os valores obtidos na competição.

Em situação de competição, a distância percorrida em função da posição ocupada pelos jogadores no campo pode variar significativamente $(1,5,12,17$,
$19,23)$ em função do estilo de jogo praticado $(3,17$, 20), do nível competitivo (2), das condições de envolvimento $(14,15,17)$, das capacidades físicas dos jogadores (6), das condições ambientais, do espaço de competição (20) e em função dos diferentes momentos da época desportiva. Contudo, esta variável pode ser considerada uma medida de produção de trabalho mecânico directamente relacionada com o gasto de energia $(12,14)$.

Na generalidade, os resultados da distância total percorrida em competição obtidos no presente estudo foram ligeiramente superiores aos referidos na litera- 
tura, facto que poderá ser indicador de maior intensidade do esforço realizado, mas que também poderá ser explicado pelas características da amostra. De facto, é provável que os jovens futebolistas ao longo do jogo e do treino apresentem mais dificuldades para decidir que posição ocupar no campo. As correcções posteriores destas decisões menos acertadas podem implicar um aumento da distância total percorrida. Quando nos centramos especificamente na distância percorrida por posição, verificamos que a maioria dos estudos realizados em competições refere que a maior distância é percorrida pelos jogadores do meio-campo e a menor pelos defesas centrais e avançados, pese embora estes últimos sejam mais flexíveis $(1,7,16,22)$. Os resultados obtidos no presente estudo confirmaram esta tendência para o contexto que foi estudado. Estas diferenças podem decorrer da função de ligação entre o ataque e a defesa que é característica destes jogadores, da quantidade de actividade desenvolvida a baixa intensidade, de limitações tácticas, dos próprios requerimentos da posição que exige uma maior movimentação, ou ainda, de uma condição física (capacidade aeróbia) superior aos jogadores de outras posições $(16,22)$.

Se no contexto da competição está disponível um conjunto alargado de estudos, no contexto do treino o mesmo não acontece. $\mathrm{O}$ único estudo que encontrámos no contexto do treino foi realizado por Luhtanen (10) e aponta valores médios de distância percorrida de $12 \mathrm{~km}$, sem no entanto referir a posição dos jogadores analisados, nem tão pouco a metodologia utilizada para recolher estes dados. No entanto, o autor refere que a principal diferença encontrada na comparação foi que as sessões de treino incluem mais intensidade resultante da prática de jogos reduzidos. Nos resultados obtidos no presente estudo, os valores da distância percorrida foram inferiores (entre $1700 \mathrm{~m}$ e $3400 \mathrm{~m}$ ), relativamente ao estudo de Luhtanen (10). No confronto entre os valores obtidos no treino e na competição, os resultados evidenciaram distâncias percorridas nas sessões de treino inferiores para todas as posições (os atletas percorreram aproximadamente menos $4 \mathrm{~km}$ ). Na comparação entre $1^{\mathrm{a}}$ e $2^{\mathrm{a}}$ parte, a literatura apresenta para o contexto do jogo um decréscimo de $5 \%$ $9 \%(4,20)$. Nos resultados do presente estudo, este valor foi de $12 \%$. Esta diminuição da distância total percorrida pode ter a sua origem em factores como a depleção do glicogénio muscular, intensidade da corrida, condições ambientais e estilo de jogo $(4,20)$. De todas as formas, Withers et al. (24) sugerem que a distância percorrida entre as duas partes do jogo sofre uma redução muito acentuada.

Todos os jogadores, à excepção do DC, percorreram maior distância na $2^{\mathrm{a}}$ parte do treino comparativamente à primeira. Assim, tal como já se tinha verificado para a distância total percorrida, em ambos os contextos foi o MC que percorreu a maior distância na $2^{\text {a }}$ parte. No que se refere a este facto, a literatura aponta que o consumo máximo de oxigénio está relacionado com a distância percorrida num jogo, e que os valores mais elevados são encontrados nos médios-centro $(12,23)$. Mais ainda, os jogadores com valores mais elevados nesta variável não apresentam decréscimos significativos nos valores da distância percorrida na $2^{\mathrm{a}}$ parte (12). Aliás, apesar das posições ocupadas serem mais flexíveis no Futebol contemporâneo, os jogadores MC apresentam uma elevada capacidade de manter o esforço muito próximo do limiar anaeróbio (20), com valores de consumo máximo de oxigénio próximos dos 75\% (14). Esta capacidade superior dos MC parece estar relacionada com a maior distância que os mesmos percorrem a baixa intensidade $(1,14,23)$. Parece então, que o impacto de um nível de condição física mais elevado é especialmente evidente nos últimos períodos do treino e das competições.

A intensidade com que um jogador executa as acções no jogo depende da forma como as equipas jogam (sistema, estilo e modelo de jogo) e da forma como condicionam o ritmo de jogo (9). No entanto, os jogadores de nível competitivo mais elevado empregam uma maior percentagem do tempo total de jogo percorrido a uma velocidade maximal $(1,12,14,15$, 23). Luhtanen (10) refere que os valores mais elevados de velocidade tendem a ser encontrados nos avançados. Para o PL, os resultados do presente estudo apresentaram um valor médio de velocidade de $3,6 \mathrm{~m} / \mathrm{s}$, que parece bem superior aos $1,99 \mathrm{~m} / \mathrm{s}$ referidos como o valor médio de velocidade durante um jogo (20). É provável que a explicação para este facto resida em diferenças metodológicas. Por outro lado e analisando agora a velocidade máxima, o estudo de Ohashi et al. (11) em contexto de competição 
identificou valores de $9 \mathrm{~m} / \mathrm{s}$, obtidos por um avançado de uma equipa profissional do Japão. Estes valores coincidem com o limite máximo apresentado por Shephard (20) e, apesar das diferenças metodológicas, foram confirmados nos resultados obtidos pelo PL avaliado no presente estudo (o valor máximo foi $10,9 \mathrm{~m} / \mathrm{s}$ ). No contexto do treino não encontrámos na literatura estudos disponíveis que pudessem conduzir a qualquer comparação de resultados.

Os valores da velocidade aumentaram da $1^{\mathrm{a}}$ para a $2^{\mathrm{a}}$ parte do treino, enquanto que na competição ocorreu o contrário, a velocidade média foi maior na primeira parte, i.e., provavelmente começou a notar-se uma quebra no trabalho realizado à medida que se aproxima o final do jogo $(13,16)$.

No contexto do treino, a subida dos valores médios da velocidade pode expressar um aumento da velocidade de execução dos exercícios, devido à metodologia utilizada na elaboração das sessões de treino, onde tradicionalmente no final das sessões se realizam situações de jogo, desde o espaço reduzido, meio campo e em campo inteiro. No entanto, este tipo de estruturação parece inverso ao que se identificou na competição, facto que pode tornar as sessões de treino menos específicas.

Em suma, durante os três microciclos de trabalho que foram analisados e face à metodologia empregue e ao tratamento que foi realizado aos resultados, pensamos ser possível concluir que existiram diferenças entre a actividade motora (nas variáveis distância total percorrida, velocidade e tempo) dos três jovens futebolistas que actuaram em posições distintas no treino e na competição. Desta forma, os resultados sugerem que o jogo de Futebol solicitou aos jogadores componentes da aptidão física diferentes das solicitadas nas sessões de treino e como tal o treino realizado nas semanas de trabalho foi menos específico.

\author{
CORRESPONDÊNCIA \\ Pedro Miguel Faria Caixinha \\ Rua da Ilha da Madeira, $2-2^{\circ} \mathrm{A}$ \\ 7800-461 Beja \\ Portugal \\ pcaixinha@netvisao.pt
}




\section{BIBLIOGRAFIA}

1 Bangsbo J, Norregaard L, Thorso F (1991). Activity profile of competition soccer. Can J Sport Sci 16: 110-116.

2 Bangsbo J (1993). The Physiology of Soccer - with special reference to intense intermittent exercise. Copenhagen: August Krogh Institute. University of Copenhagen.

3 Bangsbo J, Lindquist $\mathrm{F}$ (1992). Accumulated $\mathrm{O}_{2}$ deficit during intense exercise and muscule characteristics of elite athletes. Int J Sports Med 8: 114-117.

4 Bangsbo J (1994). Physiology of soccer - with special reference to intense intermittent exercise. Acta Physiol Scand 151: 619-612.

5 Castagna C, D' Ottavio S (1999). Activity profile of young soccer players during match-play. J Sports Sci 18: 826-827.

6 Di Salvo V (2001). Training of Elite Soccer Players according to their Positional Roles. Dissertação de Doutoramento. Faculdade de Motricidade Humana, Universidade Técnica de Lisboa, Portugal.

7 Ekblom B (1986). Applied physiology of soccer. Int J Sports Med 3: 50-60.

8 Fernandes O, Caixinha P (2003). A New Method in TimeMotion Analysis in Soccer Training and Competition. In Book of Abstracts. Science e Football, $5^{\text {th }}$ World Congress. Lisboa: Editorial Gymnos, 270-271.

9 Garganta J (1997). Modelação táctica do jogo de futebol. Estudo da organização da fase ofensiva em equipas de alto rendimento. Dissertação de Doutoramento. Faculdade de Ciências do Desporto e de Educação Física, Universidade do Porto, Portugal.

10 Luhtanen P (1994). Biomechanical aspects of soccer performance. In: B.Ekblom (ed.). Football. Oxford: Blackwell Scientific. 59-77.

11 Ohashi J, Togari H, Isokawa M, Suzuki S (1999). Measuring Movement Speeds ans Distances Covered during Soccer Match-Play. In: T. Reilly, A. Less, K. Davids e W. Murphy (Eds.). Science and Football. Proceedings of the First World Congress of Science and Football. Liverpool, 1987. London - New York: E. e F.N. Spon., 329-333.

12 Reilly T, Thomas V (1976). A motion analysis of work-rate in different positional roles in professional football matchplay. J Hum Mov Studies 2: 87-97.

13 Reilly T (1994). Soccer - Motion characteristics. In: B. Ekblom (Ed.) Handbook of Sports Medicine and Science: Football (Soccer). London: Blackwell Scientific Publications, 31-42.

14 Reilly T (1996). Motion analysis and physiological demands. In: T. Reilly (Ed.) Science and Soccer. London: EeF.N. Spon, 65-81.

15 Reilly T (1997). Energetics of high-intensity exercise (soccer) with particular reference to fatigue. J. Sports Sci 15: 257-263.

16 Reilly T, Drust B, Rienzi E (1998). Analysis of work rate in soccer. Sports Exer Inj 4: 151-155.

17 Reilly T, Drust B, Cable N (1999). Metabolic and physiological responses to a laboratory-based soccer-specific intermitent protocol on a non-monitorized treadmill. J Sports Sci 18: 811-813.

18 Reilly T, Bangsbo J, Franks A (2000). Anthropometric and physiological predispositions for elite soccer. J Sports Sci 18: 669-683.

19 Sampaio A (2000). O Poder Discriminatório das Estatísticas do Jogo de Basquetebol: Novos Caminhos
Metodológicos de Análise. Dissertação de Doutoramento, Universidade de Trás-os-Montes e Alto Douro, Vila Real, Portugal.

20 Shephard R (1999). Biology and medicine of soccer: an update. J Sports Sci 18: 757-786.

21 Smaros G (1980). Energy usage during football match. In T. Reilly, A. Less, K. Davids e W. Murphy (Eds.). Proceedings of $1^{\text {st }}$ Internacional Proceedings of the First World Congress of Science and Football. Liverpool, 1987. LondonNew York: E. e F.N. Spon., 337-339.

22 Soares J, Oliveira J, Magalhães J, Rebelo A, Duarte J, Gonçalves J (1998). The endurance capacity of soccer players evaluated by the yo-yo intermitent endurance test. In Notational Analysis of Sport. IV World Congress. Porto: FCDEF-UP, 98-100.

23 Tumilty D. (1993). Physiological characteristics of elite soccer players. J Sports Med 16: 80-96.

24 Withers R, Maricic Z, Wasilewseki S Kelly L (1982). Match analysis of Australian professional soccer players. J. Hum Mov Stud 8: 159-176. 